\title{
MAXWELL'S LAW OF DISTRIBUTION OF VELOCITIES AND THE PRINCIPLE OF PROJECTIVE COVARIANCE.
}

\author{
By I. MAIzLISH.
}

SYNopsis.

Derivation of Maxwell's Law of Distribution of Velocities with the Aid of the Principle of Projective Covariance.-In the ordinary derivation of this law there occurs a functional equation of the form: $g\left(x_{1}\right) g\left(x_{2}\right) g\left(x_{3}\right) g\left(x_{4}\right)=\psi\left(x_{1}^{2}+x_{2}{ }^{2}+x_{3}{ }^{2}+x_{4}{ }^{2}\right)$, whose solution is usually obtained on the supposition that the functions are differentiable. In this paper the solution is obtained without making use of the assumption, by applying the author's principle of projective covariance.

I. In the derivation of Maxwell's law of the distribution of velocities among a large number of molecules there occurs a certain functional equation whose solution is usually obtained by differentiation. The assumptions on which the solution is based are:

I. The functions occurring in the functional equation are continuous, single-valued, and real;

II. The independent variables are real;

III. The functions are differentiable.

2. Now it is known that a function may be continuous and yet not have a derivative. It is therefore desirable to inquire whether the functional equation can be solved without making use of assumption III. The object of this paper is to indicate a method of doing this by making use of the Principle of Projective Covariance. The problem will be generalized somewhat in that we shall consider it as a problem in a 4-dimensional space $\left(S_{4}\right)$.

3. Consider, then, an $S_{4}$ and let $x_{1}, x_{2}, x_{3}, x_{4}$ be the rectangular coördinates of a point in this space. If we are dealing with a gas containing a large number $(N)$ of molecules per unit volume, the position of any molecule will be known as soon as we know the values of $x_{1}, x_{2}, x_{3}, x_{4}$ appropriate to this molecule. Each molecule may therefore be represented by a single point in $S_{4}$. The points which are occupied by molecules will be called molecular-points. Let us now find the number of molecularpoints for which

$$
x_{i} \text { lies between } x_{i} \text { and } x_{i}+d x_{i}(i=\mathrm{I}, 2,3,4) .
$$

4. Let $N g\left(x_{i}\right) d x_{i}$ be the number of molecular-points for which $x_{i}$ lies between $x_{i}$ and $x_{i}+d x_{i} ; i=\mathrm{I}, 2,3,4$. The same function, $g$, is used 
for $i=\mathrm{I}, 2,3,4$, on account of the isotropy of $S_{4}$. If we now assume that $x_{1}, x_{2}, x_{3}, x_{4}$ are independent of each other, the number of molecularpoints for which $x_{1}, x_{2}, x_{3}, x_{4}$ lie within the range $(R)$ is

$$
\Delta_{4} N d x_{1} d x_{2} d x_{3} d x_{4}=N g\left(x_{1}\right) g\left(x_{2}\right) g\left(x_{3}\right) g\left(x_{4}\right) d x_{1} d x_{2} d x_{3} d x_{4} ;
$$

and, therefore, the number per unit volume is

$$
\Delta_{4} N=N g\left(x_{1}\right) g\left(x_{2}\right) g\left(x_{3}\right) g\left(x_{4}\right) .
$$

The rectangular axes $x_{1}, x_{2}, x_{3}, x_{4}$ are, however, entirely arbitrary. Equation (2) must therefore be a function of the radius vector only; that is, we must have

$$
g\left(x_{1}\right) g\left(x_{2}\right) g\left(x_{3}\right) g\left(x_{4}\right)=\psi\left(x_{1}^{2}+x_{2}{ }^{2}+x_{3}{ }^{2}+x_{4}^{2}\right) .
$$

This is the functional equation the solution of which will be obtained without making use of assumption III. We shall restrict our interest solely to the continuous solution of $(A)$.

5. In order to see more clearly how equation $(A)$ follows from the assumptions made, we will prove the following

THEOREM: A necessary and sufficient condition in order that a function $F\left(x_{1}, x_{2}, x_{3}, x_{4}\right)$ be invariant under any orthogonal transformation is that it be a function of the radius vector only; that is, that

$$
F\left(x_{1}, x_{2}, x_{3}, x_{4}\right)=\psi\left(x_{1}^{2}+x_{2}{ }^{2}+x_{3}{ }^{2}+x_{4}^{2}\right) .
$$

It will then follow that a necessary and sufficient condition that

$$
g\left(x_{1}\right) g\left(x_{2}\right) g\left(x_{3}\right) g\left(x_{4}\right)
$$

be invariant under any such transformation is that it be a function of the radius vector only.

Proof: (a) The condition is sufficient. For let $y_{1}, y_{2}, y_{3}, y_{4}$ be the new variables, and

$$
\left|\begin{array}{cccc}
a_{11} & a_{12} & a_{13} & a_{14} \\
a_{21} & a_{22} & a_{23} & a_{24} \\
a_{31} & a_{32} & a_{33} & a_{34} \\
a_{41} & a_{42} & a_{43} & a_{44}
\end{array}\right|
$$

the matrix of the transformation. By hypothesis,

$$
\begin{aligned}
F\left(x_{1}, x_{2}, x_{3}, x_{4}\right) & =\psi\left(x_{1}{ }^{2}+x_{2}{ }^{2}+x_{3}{ }^{2}+x_{4}{ }^{2}\right), \\
& =\psi\left(y_{1}{ }^{2}+y_{2}{ }^{2}+y_{3}{ }^{2}+y_{4}^{2}\right),
\end{aligned}
$$

because an orthogonal transformation is defined as a linear transformation such that

$$
x_{1}^{2}+x_{2}{ }^{2}+x_{3}{ }^{2}+x_{4}{ }^{2} \equiv y_{1}{ }^{2}+y_{2}{ }^{2}+y_{3}{ }^{2}+y_{4}{ }^{2} .
$$


By substitution,

and

$$
\psi\left(y_{1}^{2}+y_{2}^{2}+y_{3}^{2}+y_{4}^{2}\right)=F\left(y_{1}, y_{2}, y_{3}, y_{4}\right) ;
$$

$$
F\left(x_{1}, x_{2}, x_{3}, x_{4}\right) \equiv F\left(y_{1}, y_{2}, y_{3}, y_{4}\right) .
$$

(b) The condition is necessary. For we have

$$
\begin{aligned}
F\left(x_{1}, x_{2}, x_{3}, x_{4}\right) & \equiv F\left(y_{1}, y_{2}, y_{3}, y_{4}\right) \\
& \equiv F\left(\sum_{k=1}^{4} a_{1 k} x_{k}, \sum_{k=1}^{4} a_{2 k} x_{k}, \sum_{k=1}^{4} a_{3 k} x_{k}, \sum_{k=1}^{4} a_{4 k} x_{k}\right) .
\end{aligned}
$$

In the last equation place $x_{2}=x_{3}=x_{4}=0$. The result is

$$
F\left(x_{1}, \mathrm{o}, \mathrm{o}, \mathrm{o}\right) \equiv F\left(a_{11} x_{1}, a_{21} x_{1}, a_{31} x_{1}, a_{41} x_{1}\right) .
$$

Thus, $F$ must be of such a form as to be independent of $a_{11}, a_{21}, a_{31}$, and $a_{41}$. Since, however, $a_{11}, a_{21}, a_{31}$, and $a_{41}$ cannot all be equal to I, and, moreover, since the only condition existing between them is

$$
a_{11}^{2}+a_{21}^{2}+a_{31}^{2}+a_{41}^{2}=\mathrm{I},
$$

we see that this must be the form in which they enter in $F$. This will in general be the case if and only if

$$
F\left(a_{11} x_{1}, a_{21} x_{1}, a_{31} x_{1}, a_{41} x_{1}\right) \equiv \psi\left[\left(a_{11} x_{1}\right)^{2}+\left(a_{21} x_{1}\right)^{2}+\left(a_{31} x_{1}\right)^{2}+\left(a_{41} x_{1}\right)^{2}\right] .
$$

This is to be true for all values of $a_{11} x_{1}, a_{21} x_{1}, a_{31} x_{1}$ and $a_{41} x_{1}$. Hence, if we replace $a_{k 1} x_{1}$ by

$$
a_{k 1} x_{1}+a_{k 2} x_{2}+a_{k 3} x_{3}+a_{k 4} x_{4}, \quad k=\mathrm{I}, 2,3,4 ;
$$

the result is that

$$
\begin{aligned}
F\left(\sum_{k=1}^{4} a_{1 k} x_{k}, \sum_{k=1}^{4} a_{2 k} x_{k}, \sum_{k=1}^{4} a_{3 k} x_{k}, \sum_{k=1}^{4} a_{4 k} x_{k}\right) \\
=\psi\left[\left(\sum_{k=1}^{4} a_{1 k} x_{k}\right)^{2}+\left(\sum_{k=1}^{4} a_{2 k} x_{k}\right)^{2}+\left(\sum_{k=1}^{4} a_{3 k} x_{k}\right)^{2}+\left(\sum_{k=1}^{4} a_{4 k} x_{k}\right)^{2}\right]
\end{aligned}
$$

that is,

$$
\begin{aligned}
F\left(x_{1}, x_{2}, x_{3}, x_{4}\right) & \equiv F\left(y_{1}, y_{2}, y_{3}, y_{4}\right) \\
& =\psi\left(y_{1}{ }^{2}+y_{2}{ }^{2}+y_{3}{ }^{2}+y_{4}^{2}\right) .
\end{aligned}
$$

But, in virtue of the relations existing between the coefficients of the matrix $(M)$, it follows immediately that

Therefore,

$$
\psi\left(y_{1}^{2}+y_{2}^{2}+y_{3}^{2}+y_{4}^{2}\right) \equiv \psi\left(x_{1}^{2}+x_{2}^{2}+x_{3}^{2}+x_{4}^{2}\right) .
$$

$$
F\left(x_{1}, x_{2}, x_{3} ; x_{4}\right)=\psi\left(x_{1}^{2}+x_{2}^{2}+x_{3}^{2}+x_{4}^{2}\right) .
$$

The theorem is therefore proved. 
6. The Principle of Projective Covariance ${ }^{1}$ enables us to obtain the solution of the functional equation $(A)$ with ease. This Principle asserts that the equations occurring in Physics are covariant under the transformation:

$$
\begin{aligned}
& \xi_{1}=G(\sigma, r) x_{1}, \\
& \xi_{2}=G(\sigma, r) x_{2}, \\
& \xi_{3}=G(\sigma, r) x_{3}, \\
& \xi_{4}=G(\sigma, r) x_{4},
\end{aligned}
$$

where $G$ is an arbitrary function of the distance $(r)$ of any point from the origin of a system of coördinates in $S_{4}$, and an arbitrary constant $\sigma$; and $x_{1}, x_{2}, x_{3}, x_{4}$ are the Minkowski variables. To fix our ideas, consider the following special form of (3):

$$
\begin{aligned}
& \xi_{1}=n^{\sigma-r^{2}} x_{1}, \\
& \xi_{2}=n^{\sigma-r^{2}} x_{2}, \\
& \xi_{3}=n^{\sigma-r^{2}} x_{3}, \\
& \xi_{4}=n^{\sigma-r^{2}} x_{4},
\end{aligned}
$$

where $n$ is any number greater than $\mathrm{I}$. We have

$$
g\left(x_{1}\right) g\left(x_{2}\right) g\left(x_{3}\right) g\left(x_{4}\right)=\psi\left(x_{1}{ }^{2}+x_{2}{ }^{2}+x_{3}{ }^{2}+x_{4}{ }^{2}\right) .
$$

By the Principle of Projective Covariance, we also have

$$
g\left(\xi_{1}\right) g\left(\xi_{2}\right) g\left(\xi_{3}\right) g\left(\xi_{4}\right)=\psi\left(\xi_{1}{ }^{2}+\xi_{2}{ }^{2}+\xi_{3}{ }^{2}+\xi_{4}{ }^{2}\right) .
$$

In equation $(A)$ set $x_{2}=x_{3}=x_{4}=0$, and in equation $(B)$ set $\xi_{2}=\xi_{3}=$ $\xi_{4}=$ o. The result is

and

$$
g^{3}(\mathrm{o}) g\left(x_{1}\right)=\psi\left(x_{1}^{2}\right),
$$

$$
g^{3}(\mathrm{o}) g\left(\xi_{1}\right)=\psi\left(\xi_{1}^{2}\right) .
$$

Hence, since, from physical considerations, $g(0)$ exists,

$$
\frac{\psi\left(x_{1}^{2}\right)}{g\left(x_{1}\right)}=\frac{\psi\left(\xi_{1}^{2}\right)}{g\left(\xi_{1}\right)} .
$$

7. Now $\xi_{1}$ may be looked upon as an independent variable. For, despite the fact that $x_{1}$ and $\xi_{1}$ are connected by the transformation (4), the value of $\xi_{1}$ is not determined by $x_{1}$ alone: it also depends on $x_{2}$, $x_{3}$, and $x_{4}$. But then, the left-hand side of equation (5) is a function of $x_{1}$ only, and the right-hand side is a function of $\xi_{1}$ only. Hence, equation (5) can only be true if each member of this equation is equal to a constant. Therefore,

$$
\frac{\psi\left(x_{1}^{2}\right)}{g\left(x_{1}\right)}=\text { const. }=\beta^{3}, \text { say. }
$$

1 I. Maizlish, Physical Rev., July, x92 I. 
8. In equation $(A)$ set $x_{2}=x_{3}=x_{4}=0$. The result is

$$
g\left(x_{1}\right) g^{3}(\mathrm{o})=\psi\left(x_{1}^{2}\right) ;
$$

from which, by equation (6), we get

$$
g^{3}(0) \frac{\psi\left(x_{1}^{2}\right)}{\beta^{3}}=\psi\left(x_{1}^{2}\right)
$$

that is,

$$
g(0)=\beta .
$$

Moreover, from equation $(A)$ it is easily seen that

that is,

$$
g(0) g(0) g\left(x_{1}\right) g\left(x_{2}\right)=\psi\left(x_{1}{ }^{2}+x_{2}{ }^{2}\right),
$$

$$
\beta^{2} g\left(x_{1}\right) g\left(x_{2}\right)=\psi\left(x_{1}^{2}+x_{2}^{2}\right) .
$$

Multiply each member of equation (8) by $\beta^{4}$. Thus,

that is,

$$
\beta^{3} g\left(x_{1}\right) \beta^{3} g\left(x_{2}\right)=\beta^{4} \psi\left(x_{1}^{2}+x_{2}{ }^{2}\right),
$$

$$
\psi\left(x_{1}^{2}\right) \psi\left(x_{2}^{2}\right)=\beta^{4} \psi\left(x_{1}^{2}+x_{2}^{2}\right)
$$

by substitution from (6). Now divide each member of (9) by $\beta^{8}$.

The result is

$$
\frac{\psi\left(x_{1}^{2}\right)}{\beta^{4}} \cdot \frac{\psi\left(x_{2}^{2}\right)}{\beta^{4}}=\frac{\psi\left(x_{1}^{2}+x_{2}^{2}\right)}{\beta^{4}} ;
$$

and, on placing

this becomes

$$
\varphi(x) \equiv \frac{\psi(x)}{\beta^{4}},
$$

$$
\varphi\left(x_{1}^{2}\right) \varphi\left(x_{2}^{2}\right)=\varphi\left(x_{1}^{2}+x_{2}^{2}\right) .
$$

The last equation may be written

$$
\varphi(x) \varphi(y)=\varphi(x+y),
$$

by placing $x=x_{1}^{2}$, and $y=x_{2}{ }^{2}$.

9. Now the functional equation (II) has been solved by Cauchy, ${ }^{1}$ Darboux, ${ }^{2}$ and others. Assuming that $\varphi$ is continuous, single-valued, and real; and that $x$ and $y$ are real, Cauchy has shown that the only (continuous) solution of (II) is

$$
\varphi(x)=a^{x},
$$

where $a$ is an arbitrary constant. But these assumptions are identical with those we made at the beginning. Hence, from eqs. (Io) and (II) we get

$$
\varphi\left(x_{1}^{2}\right)=a^{x^{2}} .
$$

${ }^{1}$ Cauchy, Euvres (2), 3 (1897), p. 98.

${ }^{2}$ Darboux, Math. Ann., Bd. I7, I880, p. $5^{6}$. 
In view of eqs. (6) and $\left(9^{1}\right)$ we now have

Thus, in general,

$$
\begin{gathered}
a^{x_{1}^{2}}=\varphi\left(x_{1}^{2}\right)=\frac{\psi\left(x_{1}^{2}\right)}{\beta^{4}}=\frac{\beta^{3} g\left(x_{1}\right)}{\beta^{4}} \\
\therefore g\left(x_{1}\right)=\beta a^{x^{2}} . \\
g\left(x_{i}\right)=\beta a^{x^{2}}, \quad(i=\mathrm{I}, 2,3,4) .
\end{gathered}
$$

I0. It follows that the number of molecular-points for which $x_{1}, x_{2}$, $x_{3}, x_{4}$ lie within the range $(R)$ is, by (I) and (I 3 ),

$$
\Delta_{4} N d x_{1} d x_{2} d x_{3} d x_{4}=N \beta^{4} a^{\left[x_{1}^{2}+x_{2}^{2}+x_{3}^{2}+x_{4}^{2}\right]} d x_{1} d x_{2} d x_{3} d x_{4} ;
$$

and the number per unit volume is therefore:

$$
\Delta_{4} N=N \beta^{4} a^{\left[x_{1}^{2}+x_{2}^{2}+x_{3}^{2}+x_{4}^{2}\right]} .
$$

The constant $a$ is arbitrary: we may therefore put it equal to $e^{-h}$, where $e$ is the Napierean base, $h$ is a constant, and the minus sign is prefixed to the $h$ for otherwise $\Delta_{4} N$ would increase indefinitely with $\left(x_{1}{ }^{2}+x_{2}{ }^{2}\right.$ $+x_{3}^{2}+x_{4}^{2}$ ). Thus, equation (14) may be written as

$$
\Delta_{4} N=N \beta^{4} e^{-h\left[x_{1}^{2}+x_{2}^{2}+x_{3}^{2}+x_{4}^{2}\right]} .
$$

I I. The solution obtained is a generalization of Maxwell's. By putting $x_{4}=0$, and remembering that $g(0)=\beta$, equation (I5) reduces to

$$
\Delta_{3} N=N \beta^{3} e^{-h\left[x_{1}^{2}+x_{2}^{2}+x_{3}^{2}\right]}
$$

I2. We may regard $x_{1}, x_{2}, x_{3}$ as any set of rectangular axes in a space of three dimensions $\left(S_{3}\right)$. Take these axes as the components of a velocity $c$ defined by

where

$$
c^{2}=u^{2}+v^{2}+w^{2}
$$

and

$$
\begin{aligned}
& x_{1} \equiv u \\
& x_{2} \equiv v,
\end{aligned}
$$

$$
x_{3} \equiv w .
$$

Making this substitution, (I6) becomes

$$
\Delta_{3} N=N \beta^{3} e^{-h^{\left[u^{2}+v^{2}+w^{2}\right]}} .
$$

13. The number of molecules for which $u, v, w$ lie within the range $u$ and $u+d u$, $v$ and $v+d v$, $w$ and $w+d w$, respectively, is now seen to be

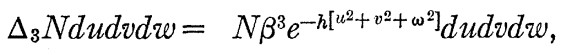

which is the solution given ${ }^{1}$ by Maxwell.

1 Maxwell, Collected Papers, Vol. I., p. 38r. 
I4. Thus, provided we justify every step in the process of arriving at equation $(A)$, and restrict our interest to the continuous solution of this equation, under the specified conditions, the solution (solutions) depends (depend) on that (those) of

$$
\varphi(x) \varphi(y)=\varphi(x+y) .
$$

But under the same restrictions as those specified at the beginning of this paper, there is only one solution of $(C)$. Hence, there is only one solution of $(A)$, and, by the application of the Principle of Projective Covariance, this has been shown to be

$$
g(x)=\beta e^{-h x 2} .
$$

I am grateful to Professor R. W. Brink, of the Department of Mathematics, for examining the manuscript.

Dept. of Physics,

The University of Minnesota,

MINNEAPOLIS,

March 23, 1922. 